\title{
Targeted Path Scanning: An Emerging Method for Recording Fast Changing Network Dynamics across Large Distances
}

Smeal RM ${ }^{1 *}$, Economo $\mathrm{MN}^{2}$, Lillis KP ${ }^{3}$, Wilcox KS ${ }^{1}$ and White $\mathrm{JA}^{2}$

${ }^{1}$ Department of Pharmacology and Toxicology, University of Utah, USA

${ }^{2}$ Department of Bioengineering, University of Utah, USA

${ }^{3}$ Department of Neurology, Massachusetts General Hospital, USA

\begin{abstract}
Attention is being increasingly focused on the dynamical behavior of large networks of neurons and astrocytes and the changes in these dynamics that occur during the progression of diseases like epilepsy. Recording from large numbers of identified cell types has been traditionally difficult, but the advent of fluorescent indicators capable of detecting changes in the internal calcium levels of cells has led to the ability to visually record the activity of large numbers of cells. However, for most imaging techniques the temporal resolution is sharply limited by the time it takes lasers to traverse the typical raster scan. As network dynamics can evolve quite rapidly, this is a serious limitation. The present paper describes the Targeted Path Scan technique, which dramatically increases the scanning frequency by allowing the user selection of trajectories through cells of interest. TPS is discussed in the context of a study of altered network dynamics in a common rat model of epilepsy. In this study, traveling waves of calcium transients that were frequently encountered in astrocytes imaged in brain slices obtained from control rats were dramatically reduced in astrocytes imaged in brain slices obtained from rats that had experienced status epilepticus. The speed of these traveling waves would have made them impossible to identify using traditional scanning techniques.
\end{abstract}

Keywords: Neurons; Astrocytes; Calcium imaging; Hippocampus; Targeted path scan technique

\section{Introduction}

The dynamic formation of cell assemblies defined by their mutual patterns of activity is thought to be fundamental for many neural processes [1-3]. Additionally, neurons can interact with glia, particularly astrocytes, potentially forming complex and poorly understood neuron-astrocyte dynamical systems [4-8]. Distinct changes in patterns of network activity have been correlated with a number of diseases including Parkinson's disease [9-11] schizophrenia [12] autism [13], and epilepsy [14-16]. While much of the work to date has focused on synchronous activity, other dynamic network patterns have received increasing attention such as traveling waves, which are prominent in astrocytic networks [7,17-19]. As the astrocytic traveling waves can be quite fast [7], it is important to have a fast scanning technique to distinguish them from purely synchronous network activity. The determination of such complicated network activity requires experimental techniques that can record activity from a large number of cells over distances spanning the size of networks, and to accomplish this task quickly enough to capture the relevant dynamics. As calcium levels in cells are correlated with activity in both neurons and astrocytes, the imaging of fluorescent calcium indicators has the potential of satisfying the aforementioned requirements. However, to date, imaging the calcium dynamics in numerous cells (10s to 100 s) over the relevant distances of at least 100 s of micrometer is quite difficult for frame rates above a few $\mathrm{Hz}$.

A technique, Targeted Path Scanning (TPS), for the imaging of calcium changes in a large number of cells over extended distances with high spatiotemporal resolution has been developed by Lillis et al. [20] TPS is an extension of previous studies [21,22] and uses standard two-photon microscopy hardware. TPS improves on the inherently slow nature of raster scanning for generating image sequences by allowing the selection of specific trajectories for the two-photon beam through the cells of interest. The scanning speed is further enhanced by maintaining a user-defined sampling rate within cells of interest while accelerating the beam between cells of interest, thus minimizing dead time. This technique results in scan rates up to $100 \mathrm{~s}$ of $\mathrm{Hz}$ for millimeterscale distances and maintains the superior signal-to-noise ratio of twophoton microscopy. The technique is discussed in the present paper for the application of determining changes in astrocytic network activity in brain slices prepared from rats that have experienced kainic acid (KA)-induced status epilepticus (SE), a common animal model of epilepsy that results in reactive astrogliosis and changes in astrocyte function. A primary finding in the present set of experiments is that fast traveling waves of calcium activity in astrocytes in the CA1 region of the hippocampus are less prominent in rats that experienced early life SE. Furthermore, the speed of these traveling waves would have made them difficult to distinguish from synchronous activity if traditional imaging techniques had been used. Therefore, TPS will be instrumental in identifying rapid calcium signaling events that are changed as a consequence of neural and glia pathology.

\section{Methods}

\section{Slice preparation and labeling with SR101 and Indo-1}

All experimental protocols were approved by the University of Utah Institutional Animal Care and Use Committee. Sprague-Dawley rats (P17-P20) were injected i.p with $7.5 \mathrm{mg} / \mathrm{kg}$ kainic acid and observed for

*Corresponding author: Roy M. Smeal, Department of Pharmacology and Toxicology, University of Utah, 417 Wakara Way, Suite 3211, Salt Lake City, UT 84108 USA; E-mail: roy.smeal@m.cc.utah.edu

Received November 11, 2011; Accepted December 13, 2011; Published December 30, 2011

Citation: Smeal RM, Economo MN, Lillis KP, Wilcox KS, White JA, et al. (2012) Targeted Path Scanning: An Emerging Method for Recording Fast Changing Network Dynamics across Large Distances. J Bioengineer \& Biomedical Sci S5:002. doi:10.4172/2155-9538.S5-002

Copyright: (c) 2012 Smeal RM, et al. This is an open-access article distributed under the terms of the Creative Commons Attribution License, which permits unrestricted use, distribution, and reproduction in any medium, provided the original author and source are credited. 
3 hrs following injection. Seizures were classified on the Racine scale (1-5) and animals with significant seizures, class 4-5, were selected for study [23]. Following treatment, rats were returned to their home cages with their mother. Control animals were sham injected with vehicle (0.9\% saline). After 3-5 days recovery, rats were deeply anesthetized with isoflurane and brains were rapidly dissected and placed in icecold $\left(4^{\circ} \mathrm{C}\right)$ oxygenated sucrose Ringer's solution $\left(95 \% \mathrm{O}_{2} / 5 \% \mathrm{CO}_{2}\right)$ containing (in $\mathrm{mM}$ ): 200 sucrose, $26 \mathrm{NaHCO}_{3}, 10$ glucose, $3 \mathrm{KCl}$, and $1.4 \mathrm{NaH}_{2} \mathrm{PO}_{4}$. Brains were then trimmed, and glued ventral side down to the mounting disk of a Leica VT1200 (Leica Microsystems, Wetzlar DE). Horizontal brain slices $(400 \mu \mathrm{m})$ containing both the medial entorhinal cortex and hippocampus were cut in oxygenated sucrose Ringer's solution $\left(4^{\circ} \mathrm{C}\right)$. Following sectioning, slices were transferred to a holding chamber that contained $1 \mu$ M SR101 (Sigma-Aldrich, St. Louis, MO) [24,25] in normal, oxygenated Ringer's solution $\left(34^{\circ} \mathrm{C}\right)$ containing (in $\mathrm{mM}$ ): $126 \mathrm{NaCl}, 26 \mathrm{NaHCO}_{3}, 10$ glucose, $3 \mathrm{KCl}, 1.4$ $\mathrm{NaH}_{2} \mathrm{PO}_{4}, 2 \mathrm{CaCl}_{2}$, and $2 \mathrm{MgCl}_{2}$. Following incubation in SR101 (10-12 $\mathrm{min}$ ), slices were transferred to a separate holding chamber containing oxygenated Ringer's solution without SR101 (room temperature) for $10 \mathrm{~min}$. The slices were then incubated in $100 \mu \mathrm{g} / \mathrm{ml}$ Indo - $1 \mathrm{AM}$ (Invitrogen) in ACSF containing 0.04\% Pluronic (Molecular Probes) at $38^{\circ} \mathrm{C}$ for at least $1 \mathrm{hr}$. The higher temperature (personal communication with Dr. Doug Coulter) helped with the notoriously difficult labeling of brain slices prepared from young adult rats $[26,27]$. Following bulk dye labeling the slices were incubated in Ringer's solution at room temperature for at least $30 \mathrm{~min}$ to allow for cleavage of the AM ester group thus trapping the calcium indicator in the cells.

\section{Calcium imaging}

For imaging, slices were transferred to an immersion style recording chamber (Warner Instruments) and perfused with oxygenated Ringer's at $32^{\circ} \mathrm{C}$. Calcium imaging was performed using a custom-built twophoton microscope [20] built around a mode-locked Ti: Sapphire laser source emitting $140 \mathrm{fs}$ pulses at an $80 \mathrm{MHz}$ repetition rate with a wavelength adjustable from 690-1040 nm (Chameleon Ultra I; Coherent, Santa Clara, CA). The ability to adjust the laser wavelength gives us considerable flexibility in choosing fluorescent dyes. In this setup, laser power is attenuated using an electro-optic modulator (ConOptics, Danbury, CT) and scanning is accomplished using $\mathrm{x}-\mathrm{y}$ galvonometer-mounted mirrors (GSI Lumonics, Billerica, MA) controlled by custom LabView software [20]. Glial cells are identified as cells containing both Indo-1 (460-580 nm bandpass filter) and sulforhodamine 101 (600-660 $\mathrm{nm}$ bandpass filter) in an XY raster scan using a 20X $0.95 \mathrm{NA}$ water-immersion objective (Olympus, Tokyo, Japan). Fluorescently identified glial cells containing both SR101 and Indo-1 in stratum radiatum of the CA1 region of the hippocampus were selected for the TPS trajectories [20]. The CA1 region is thought to be involved in seizure generation in animal models of epilepsy and was the first region we examined using the TPS technique.

\section{Data analysis}

A fluorescence time series can be extracted from the path scan for all cells by averaging the fluorescence intensity of the pixels belonging to each cell, removing spurious exponential trends introduced by photobleaching, and calculating the change in fluorescence at each time point normalized by resting fluorescence. Additionally, the time series can be filtered to reduce noise using a 4-pole Butterworth filter with a cutoff frequency of $5 \mathrm{~Hz}$. Calcium 'spikes' in glial cells are identified as calcium transients having rapid onsets, decay time constants of $>2$ seconds, and $\Delta \mathrm{F} / \mathrm{F}$ amplitudes greater than $10 \%$. This data analysis is performed with custom-written scripts in Matlab (Math Works, Natick, MA).

\section{Results}

\section{Recording from networks of astrocytes}

The dynamics of neural networks result from complex interactions of a variety of types of neurons and glia. TPS is utilized in the present paper to specifically examine the internal calcium dynamics of astrocytes. The astrocytes were identified by double labeling the freshly prepared rat brain slices with SR101, the astrocyte specific fluorescent marker [24,25] and Indo-1 AM, a fluorescent indicator that changes level of fluorescence in response to changes in calcium concentration. An example of SR101 labeling is shown in (Figure 1A) and Indo-1 labeling in (Figure 1B). The fluorescent dyes and indicators were introduced into the tissue by incubating the tissue in solutions containing the fluorophores. This resulted in the labeling of approximately the superficial 50 microns of tissue in the 400 micrometer thick brain slices, a limitation when bulk labeling brain slices prepared from older animals, greater than postnatal day 17 [26]. The co-labeling of glial cells by these two fluorescent dyes is shown in (Figure 1C). The majority of cells are co-labeled indicating that in the conditions of dye labeling in the present work, Indo-1 preferentially,
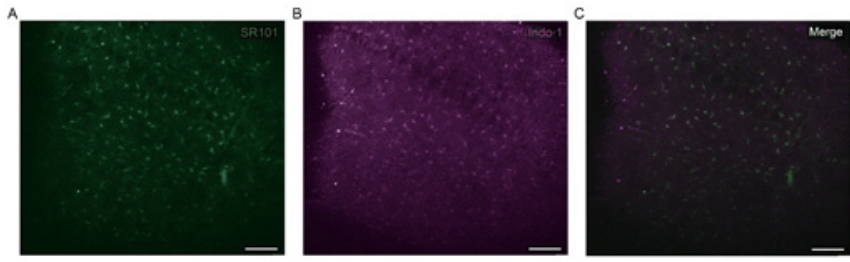

Figure 1: Networks of astrocytes were identified and loaded with calcium indicators by co-labeling cells with SR101 and Indo-1 AM. A) Two-photon image of cells labeled with the astrocyte specific marker SR101. B) Two-photon image of the same brain slice labeled with the fluorescent calcium indicator Indo-1 AM. C) A merged image displaying co-labeled astrocytes (indicated by white shading).
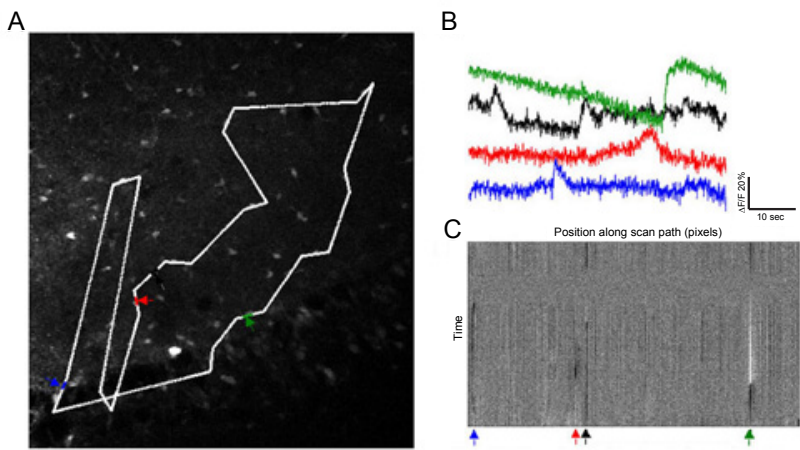

Figure 2: Spontaneous calcium transients in astrocytes recorded using TPS. A) An example TPS line scan targeting Indo-1 labeled astrocytes in the hippocampus. Colored arrows (gray, blue, red, and green) indicate the position of four different labeled cells in the chosen scan. In this case, there were few labeled neurons in the pyramidal cell layer toward the lower part of the image indicating a preference of Indo-1 for astrocytes. B) Examples of calcium transients recorded with the two-photon microscope using TPS. The four colors correspond to the astrocytes in Figure 4. C) Raw data from TPS experiment. The horizontal axis contains all the pixels of individual line scans displayed as a line. The vertical axis is time; each line corresponding to one scan and with time progressing from top to bottom. The locations of the four cells are marked with color-coded arrows at the bottom. 
but not exclusively, labeled astrocytes. The imaged network shown in (Figure 1) spans an area of approximately $600 \times 600 \mu \mathrm{m}$. This is the size of astrocytic network that has traditionally been imaged with a frame rate on the order of $1 \mathrm{~Hz}[7,28]$.

\section{Selection of the trajectory and recording calcium events}

The selection of the trajectory for the targeted path scan starts with a $\mathrm{x}-\mathrm{y}$ rastor scan of the cells of interest (Figure $2 \mathrm{~B}$ ). Software implemented in Labview [20] then allows for a point-and click selection of the trajectory with high resolution lines of interest (Figure 2A and Figure $4 \mathrm{~A}$ ) alternating with the accelerated portion of the trajectory between cells. This alternating high and low resolution between the cells of interest and the space between them, respectively, improves the frequency with which each cell is sampled, allows for greater distances to be recorded, and because of the increased time spent in the region of interest improves the signal-to-noise ratio. The path shown in (Figure 2A) is arbitrary and contains a number of less-than-optimal sharp angles. Figure $4 \mathrm{~A}$ is an example an approximately spiral path that reduces the number of sharp angles and improves the accuracy of the line path at very fast scanning speeds. The frequency with which the two-photon beam completes the selected path depends on the length of the selected path and the number of cells [20].

A time series of changes in calcium levels is calculated by averaging the fluorescence intensity of the pixels belonging to each cell and calculating the change in fluorescence at each time point normalized by resting fluorescence $(\Delta \mathrm{F} / \mathrm{F}$, Figure $2 \mathrm{~B})$. The four traces in (Figure 2B) show examples of spontaneous astrocytic calcium transients with their typical slow kinetics on the order of seconds. An example of the raw data collected using the TPS technique is shown in (Figure 2C). Each horizontal line of pixels is an unwrapped and straightened line scan trajectory, in this case the trajectory shown in Figure 2A. Time proceeds downward on the vertical axis with the increment of time defined by the time taken to complete one line scan which is dependent of the details of each targeted path. The location of four astrocytes in the unwrapped line scans are highlighted by arrows at the bottom of (Figure 2C). In this two-photon application, Indo-1 is a calcium indicator that decreases the level of fluorescence in response to an increase in internal calcium, thus the calcium transients can be identified by the darker locations in the data set. While the spontaneous calcium transients were generally quite rare in these recordings, only occurring a few times over the course of minutes, the transients did include 10 s of astrocytes per experiment. These events do not require the fast scanning capabilities of the TPS technique and will not be further explored in the present work.

\section{Fast Traveling waves require higher scan rates for accurate imaging}

A distinctive network dynamic that was frequently encountered
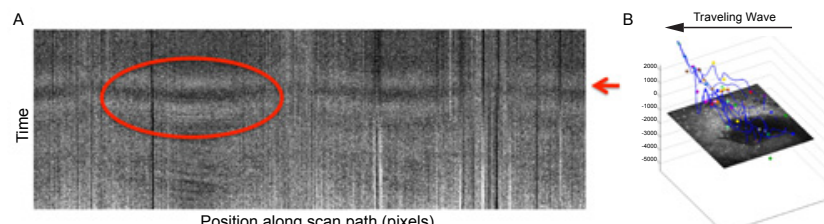

Position along scan path (pixels)

Figure 3: Traveling waves were the most prominent network dynamic observed. A) Raw data from a TPS experiment containing a traveling wave. The red oval outlines the distinctive structure of the traveling wave in these data. B) A three dimensional rendering of the trajectory over an image of the slice with the height showing relative fluorescence. The traveling wave can be seen moving from right to left in this image.
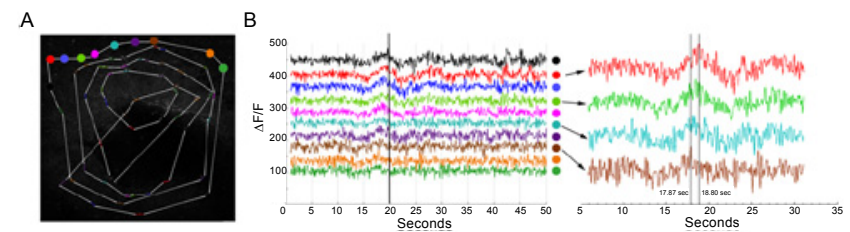

Figure 4: Traveling waves can traverse large regions of the brain slices (horizontal distance is $650 \mu \mathrm{m}$ ). A) An example TPS trajectory overlaid on a raster scan showing Indo-1 labeling. Ten cells are marked with colored circles that participated in a traveling wave. B) Fluorescent time series showing $\Delta F / F$ for the ten cells marked in A. The vertical black line is marked at the peak in the black trace (representing a minimum of calcium concentration for Indo-1) to highlight the different times of arrival of the peak at different locations.

A

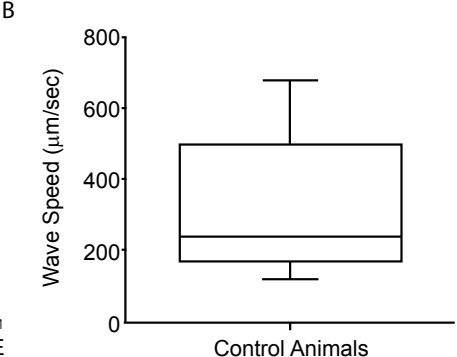

Figure 5: Traveling waves are suppressed in animals that have experienced SE. A) Bar graph showing the ratio of brain slices where a rapid traveling wave was observed to the total slices examined. There was a significant reduction in the incidence of traveling wave activity in rats that experienced $S E(P<0.05$ two-proportion test). B) Box plot of the speeds of the traveling waves (the bars show the upper and lower limits). The average speed was $309 \mu \mathrm{m} / \mathrm{sec}$.

in networks of CA1 astrocytes in brain slices prepared from young adult rats was the traveling calcium wave. An example of a traveling wave recorded from a sham-injected rat is shown in (Figure 3). The plot of unwrapped trajectories versus time is shown in (Figure 3A) and the red oval outlines the distinctive features of the traveling wave in this form of the data set. The pattern repeats to the left and right of the oval. This is simply due to the spiraling nature of the trajectory, which traverses the same region of the slice multiple times. (Figure $3 \mathrm{~B}$ ) shows a three-dimensional rendering of the trajectory over an image of the slice. The blue line is the trajectory and the colored spheres are the higher resolution regions along the trajectory overlying astrocytes. The height represents the relative fluorescence. The distinct slope of the relative fluorescence, rising from right to left in (Figure 3B), highlights the traveling wave at a particular time point. The time point for this rendering is marked by the red arrow on the data in (Figure 3A).

The characteristics of the traveling wave can be further examined by plotting the fluorescent time series from astrocytes participating in the wave (Figure 4). As shown in (Figure 4A), 10 astrocytes that participated in the wave were selected for a plot of fluorescent changes over time. These astrocytes are marked with colored circles along the targeted path and overlaid on the $\mathrm{x}-\mathrm{y}$ image that was used for cell selection. The corresponding fluorescent time series are shown in (Figure 4B). The first peak encountered occurs on the astrocyte marked with the green circle on the right side of (Figure 4A). The corresponding trace in (Figure 4B) is shown at the bottom. From right to left, the peaks in $\Delta \mathrm{F} / \mathrm{F}$ occur at later time points as the astrocytes are positioned more to the left, indicating a traveling wave from right to left in this example.

The incidence of rapid traveling waves was different in brain slices obtained from control rats versus brain slices prepared from rats that 
experienced KA-induced SE. Out of 10 slices prepared from sham injected animals 7 exhibited traveling waves (Figure 5A). However, out of 21 slices prepared from rats that experienced SE, traveling waves could be found in only 2 . These ratios were significantly different $(\mathrm{P}$ $<0.05$; two-proportion test). The speed of the traveling waves in the control animals ranged from 122 to $680 \mu \mathrm{m} / \mathrm{sec}$ and averaged $309 \mu \mathrm{m} /$ sec (Figure 5B). The speeds of the 2 waves recorded from brain slices prepared from treated animals were 334 and $230 \mu \mathrm{m} / \mathrm{sec}$, within the range of control speeds. The speed of these traveling is the same order of magnitude, but somewhat faster, than similar traveling waves observed in rat in vivo [7] also using two-photon microscopy with a limited field of view of only 10s instead of 100s of $\mu \mathrm{m}$. Waves traveling at these speeds would enter and exit fields of view of the 100s of $\mu \mathrm{m}$ squared in approximately a second making sampling rates well above $1 \mathrm{~Hz}$ crucial for distinguishing the traveling wave from true synchronous activity. The decrease in structured activity in slices from KA-treated rats may represent a good biomarker for epilepsy-induced changes in network activity. The proper characterization of network activity, traveling wave versus synchronous for example, is important for the formation and testing of hypotheses about pathological changes in network function. Important distinctions could be obscured with slower scanning rates making techniques like TPS an important tool.

\section{Discussion}

The present study used targeted path scanning (TPS) to characterize rapid calcium waves across large networks of astrocytes. We found that ultra rapid waves were only detectable as a consequence of the sampling frequency conferred by TPS and that these waves were significantly diminished in incidence in slices obtained from KA-treated rats. It is hypothesized that an important aspect of astrocyte signaling and subsequent modulation of the surrounding neural network is the pattern of calcium activity through many astrocytes in the syncytium. Indeed, recent experiments performed in vivo suggests that $\mathrm{Ca}^{2+}$ responses in astrocytes may be as fast as their neuronal counterparts, and therefore relevant during fast synaptic transmission [29]. Twophoton microscopy is a powerful visualization technique that gives excellent spatial resolution in three dimensions of fluorescent signals. However, scanning an entire two-dimensional image by moving the laser in a classic raster fashion is too slow to capture calcium transients associated with action potentials in neurons or the calcium transients in astrocytes in many cells at once. This limitation is particularly problematic because astrocytes are hypothesized to modulate neural network activity through linking more distant regions via patterned calcium activity across the astrocytic syncytium [8]. In order to test if patterned calcium activity is altered in pathological tissue, we must quickly image many cells over larger areas of tissue. Additionally, changes in patterns of calcium activity in astrocytes are hypothesized to modulate neuronal network activity. The assessment of network activity in response to astrocyte calcium changes, like the measurement of calcium fluctuations in the astrocyte syncytium, requires the speedy recording from numerous astrocytes and neurons over a spatial extent much larger that a single cell. The criteria of speedy and spatially extensive imaging are crucial for the development of a true network (as opposed to cellular) imaging technique. TPS fulfills these requirements.

In the present study utilizing TPS, networks of astrocytes imaged in the stratum radiatum of CA1 in the hippocampus were found to exhibit fast traveling waves in control tissue, but substantially fewer waves in brain slices prepared from rats that experienced SE. These waves were quite fast, ranging from 122 to $680 \mu \mathrm{m} / \mathrm{sec}$. This is even faster than similar traveling waves observed in previous work in rat in vivo that ranged from 40 to $80 \mu \mathrm{m} / \mathrm{sec}$, but imaging a much smaller field of view [7]. Astrocytic calcium waves have also been described in cultured astrocytes [17-19], in acute brain slices [30], and in studies of spreading depression $[31,32]$. The speeds of these waves ranged from 10 to $40 \mu \mathrm{m} / \mathrm{sec}$ and usually were independent of neuronal activity. The faster traveling waves of the Kuga et al. study were found to be dependent on neuronal activity [7]. The fast traveling waves in the present work are also likely dependent on neuronal network activity as the known mechanisms for propagation of traveling waves in astrocytes independent of neural activity, extracellular diffusion of ATP and IP ${ }_{3}$ diffusion through gap junctions, do not support waves this fast. It is not currently clear why astrocytes in slices obtained from KAinduced SE treated rats lacked these fast waves. Indeed, KA-induced $\mathrm{SE}$ at the young ages used in this study does not typically result in the extensive cell death that is observed in adult animals. Furthermore, in contrast to SE in adult animals, early life SE does not tend to result in the development of epilepsy, although seizure susceptibility to a second insult is dramatically increased [33]. Nevertheless, astrocytes demonstrate properties of reactive astrogliosis, with increases in expression of glial fibrillary acidic protein (GFAP) following early life seizures. Thus the astrocytes in slices from the KA-induced SE treated rats suggests that this treatment is sufficient to induce alterations in network calcium signaling.

The imaging and analysis of network activity is potentially a very powerful experimental tool for studying network pathology as changes in synchronization of neurons and glial cells have been hypothesized to be involved in a number of disorders including Parkinson's disease, autism, epilepsy, Alzheimer's disease, and schizophrenia [13,34]. Because changes in synchronization and other patterns of network behavior are seen in a variety of neurologic disorders in the absence of overt cell death, a technique recording network patterns is potentially more sensitive to changes following damage. Distinct and complex network-level dynamics precede the onset of pathological synchrony observed in patients [16], animal models of epilepsy [35], and in neuronal networks in vitro [36]. As has been shown for astrocytes previously [7] and in the present work, the speed of the astrocyte dynamics can test the limit of traditional imaging techniques making techniques like TPS important for these applications.

\section{Acknowledgments}

This work was supported by the National Institutes of Health NS069033 (JAW and KSW), RR020115 (JAW) and NS062419 (KSW). We would also like to thank the Office of the Vice President for Research, which provided a University of Utah Interdisciplinary Seed Grant (KSW and JAW). Special thanks to the ADD Program and Katherine P. Flood for outstanding technical assistance.

\section{References}

1. von der Malsburg C, Schneider W (1986) A neural cocktail-party processor. Bio Cybern 54: 29-40.

2. Fries $P$ (2005) A mechanism for cognitive dynamics: neuronal communication through neuronal coherence. Trends Cogn Sci 9: 474-480.

3. Singer W (1999) Neuronal synchrony: a versatile code for the definition of relations? Neuron 24: 49-65, 111-125.

4. Araque A, Sanzgiri RP, Parpura V, Haydon PG (1998) Calcium elevation in astrocytes causes an NMDA receptor-dependent increase in the frequency of miniature synaptic currents in cultured hippocampal neurons. J Neurosci 18 6822-6829.

5. Fellin T, Pascual O, Gobbo S, Pozzan T, Haydon PG, et al. (2004) Neuronal synchrony mediated by astrocytic glutamate through activation of extrasynaptic NMDA receptors. Neuron 43: 729-743.

6. Halassa MM, Florian C, Fellin T, Munoz JR, Lee SY, et al. (2009) Astrocytic 
Citation: Smeal RM, Economo MN, Lillis KP, Wilcox KS, White JA, et al. (2012) Targeted Path Scanning: An Emerging Method for Recording Fast Changing Network Dynamics across Large Distances. J Bioengineer \& Biomedical Sci S5:002. doi:10.4172/2155-9538.S5-002

modulation of sleep homeostasis and cognitive consequences of sleep loss. Neuron 61: 213-219.

7. Kuga N, Sasaki T, Takahara Y, Matsuki N, Ikegaya Y (2011) Large-scale calcium waves traveling through astrocytic networks in vivo. J Neurosci 31: 2607-2614.

8. Halassa MM, Haydon PG (2010) Integrated brain circuits: astrocytic networks modulate neuronal activity and behavior. Annu Rev Physiol 72: 335-355.

9. Brown P (2003) Oscillatory nature of human basal ganglia activity: relationship to the pathophysiology of Parkinson's disease. Mov Disord 18: 357-363.

10. Hammond C, Bergman H, Brown P (2007) Pathological synchronization in Parkinson's disease: networks, models and treatments. Trends Neurosci 30: 357-364

11. Murer MG, Tseng KY, Kasanetz F, Belluscio M, Riquelme LA (2002) Brain oscillations, medium spiny neurons, and dopamine. Cell Mol Neurobiol 22: 611632

12. Spencer KM, Nestor PG, Perlmutter R, Niznikiewicz MA, Klump MC, et al. (2004) Neural synchrony indexes disordered perception and cognition in schizophrenia. Proc Natl Acad Sci U S A 101: 17288-17293.

13. Uhlhaas PJ, Singer W (2006) Neural synchrony in brain disorders: relevance for cognitive dysfunctions and pathophysiology. Neuron 52: 155-168.

14. Milton J, Jung P (2003) Epilepsy as a dynamic disease. Berlin; New York: Springer. xxxii, 417 p. p.

15. Tian GF, Azmi H, Takano T, Xu Q, Peng W, et al. (2005) An astrocytic basis of epilepsy. Nat Med 11: 973-981.

16. Truccolo W, Donoghue JA, Hochberg LR, Eskandar EN, Madsen JR, et al (2011) Single-neuron dynamics in human focal epilepsy. Nat Neurosci 14: 635641

17. Cornell-Bell AH, Finkbeiner SM, Cooper MS, Smith SJ (1990) Glutamate induces calcium waves in cultured astrocytes: long-range glial signaling. Science 247: 470-473.

18. Charles AC, Merrill JE, Dirksen ER, Sanderson MJ (1991) Intercellular signaling in glial cells: calcium waves and oscillations in response to mechanical stimulation and glutamate. Neuron 6: 983-992.

19. Finkbeiner S (1992) Calcium waves in astrocytes-filling in the gaps. Neuron 8 $1101-1108$

20. Lillis KP, Eng A, White JA, Mertz J (2008) Two-photon imaging of spatially extended neuronal network dynamics with high temporal resolution. J Neurosci Methods 172: 178-184

21. Nikolenko V, Poskanzer KE, Yuste R (2007) Two-photon photostimulation and imaging of neural circuits. Nat Methods 4: 943-950.
22. Gobel W, Kampa BM, Helmchen F (2007) Imaging cellular network dynamics in three dimensions using fast 3D laser scanning. Nat Methods 4: 73-79.

23. Racine RJ (1972) Modification of seizure activity by electrical stimulation. II. Motor seizure. Electroencephalogr Clin Neurophysiol 32: 281-294.

24. Kafitz KW, Meier SD, Stephan J, Rose CR (2008) Developmental profile and properties of sulforhodamine 101--Labeled glial cells in acute brain slices of rat hippocampus. J Neurosci Methods 169: 84-92.

25. Takahashi DK, Vargas JR, Wilcox KS (2010) Increased coupling and altered glutamate transport currents in astrocytes following kainic-acid-induced status epilepticus. Neurobiol Dis 40: 573-585.

26. Peterlin ZA, Kozloski J, Mao BQ, Tsiola A, Yuste R (2000) Optical probing of neuronal circuits with calcium indicators. Proc Natl Acad Sci USA 97: 36193624.

27. Reeves AM, Shigetomi E, Khakh BS (2011) Bulk loading of calcium indicato dyes to study astrocyte physiology: key limitations and improvements using morphological maps. J Neurosci 31: 9353-9358.

28. Aguado F, Espinosa-Parrilla JF, Carmona MA, Soriano E (2002) Neuronal activity regulates correlated network properties of spontaneous calcium transients in astrocytes in situ. J Neurosci 22: 9430-9444.

29. Winship IR, Plaa N, Murphy TH (2007) Rapid astrocyte calcium signals correlate with neuronal activity and onset of the hemodynamic response in vivo. J Neurosci 27: 6268-6272.

30. Scemes E, Giaume C (2006) Astrocyte calcium waves: what they are and what they do. Glia 54: 716-725.

31. Peters O, Schipke CG, Hashimoto Y, Kettenmann H (2003) Different mechanisms promote astrocyte $\mathrm{Ca} 2+$ waves and spreading depression in the mouse neocortex. J Neurosci 23: 9888-9896.

32. Chuquet J, Hollender L, Nimchinsky EA (2007) High-resolution in vivo imaging of the neurovascular unit during spreading depression. J Neurosci 27: 40364044

33. Somera-Molina KC, Robin B, Somera CA, Anderson C, Stine C, et al. (2007) Glial activation links early-life seizures and long-term neurologic dysfunction evidence using a small molecule inhibitor of proinflammatory cytokine upregulation. Epilepsia 48: 1785-1800.

34. Vincent AJ, Gasperini R, Foa L, Small DH (2010) Astrocytes in Alzheimer's disease: emerging roles in calcium dysregulation and synaptic plasticity. J Alzheimers Dis 22: 699-714.

35. Cymerblit-Sabba A, Schiller Y (2010) Network dynamics during development of pharmacologically induced epileptic seizures in rats in vivo. J Neurosci 30: 1619-1630.

36. Cohen I, Huberfeld G, Miles R (2006) Emergence of disinhibition-induced synchrony in the CA3 region of the guinea pig hippocampus in vitro. $\mathrm{J}$ Physio 570: 583-594. 